\title{
Prediction of New Phases of Nitrogen at High Pressure from First-Principles Simulations
}

\author{
William D. Mattson, ${ }^{1, *}$ Daniel Sanchez-Portal, ${ }^{2}$ Simone Chiesa, ${ }^{1}$ and Richard M. Martin ${ }^{1}$ \\ ${ }^{1}$ Department of Physics, University of Illinois at Urbana-Champaign, 1110 W. Green Street, Urbana, Illinois 61801, USA \\ ${ }^{2}$ Centro Mixto CSIC-UPV/EHU and Donostia International Physics Center (DIPC), Apartado 1072, 20080 San Sebastian, Spain
}

(Received 20 February 2004; published 14 September 2004)

A rich variety of competing phases is predicted for nitrogen at accessible pressures, including a new metallic chainlike phase very close in energy to the previously predicted cubic gauche phase, and other phases at slightly higher energies, e.g., one with $\mathrm{N}_{2}$ and $\mathrm{N}_{6}$ units. Large energy barriers between structures can account for recent observations of metastability, and we identify a low barrier transition path from the known $\varepsilon$ phase to the chainlike metallic phase. In analogy to $\mathrm{MgB}_{2}$, the metal is anisotropic with multiple Fermi surfaces formed from $\pi$ and $\sigma$ states.

DOI: 10.1103/PhysRevLett.93.125501

The nature of nitrogen at high pressure has been studied for many decades; however, it has remained a quandary to both experimentalists and theorists. Shock wave data [1,2] have long indicated dissociation of the $\mathrm{N}_{2}$ molecules at high temperatures (above $4000 \mathrm{~K}$ ) and pressure $\sim 30 \mathrm{GPa}$, generally consistent with many theoretical calculations [3-8]. However, low temperature experiments in diamond cells have found that molecular structures persist above $100 \mathrm{GPa}$ [9], far outside the range of discrepancies for other similar materials [10,11]. Recently, the first experimental evidence of nonmolecular phases at low temperatures has been reported [12-14], apparently with an amorphous structure. The forward and backward transformations have large temperaturedependent hysteresis and possible metastability even to zero pressure [13]. In addition, new molecular phases have been observed as a function of temperature and pressure that exhibit large ranges of metastability [14].

Previous theoretical investigations have been restricted to structures that can be considered distortions of simple cubic. The first studies were initially based upon an expected analogy with phosphorus and arsenic $[3,4]$, which are semimetallic or narrow gap semiconductors, and were later extended to higher pressure by studying metallic simple cubic and a simple tetragonal phase [5]. All of these phases have energy higher than the "cubic gauche" (CG) structure proposed by Mailhiot, Yang, and McMahan [6] which remains the most stable nonmolecular structure found up to now. CG is a connected threedimensional network of threefold coordinated $\mathrm{N}$ atoms [see Fig. 2(d)], which is insulating with a large predicted [15] gap of $8.3 \mathrm{eV}$. It has low energy in both local density functional [6] and Monte Carlo [15] calculations, which, respectively, predict a pressure of $\sim 30$ and $\sim 50 \mathrm{GPa}$ for the transition from the known molecular $\varepsilon$ phase to CG. Recently, a molecular dynamics study [8] found a new metastable structure composed of $\mathrm{N}$ chains packed in a body-centered orthorhombic structure with Imma symmetry; however, it has enthalpy significantly higher than $\mathrm{CG}$ at all pressures above $15 \mathrm{GPa}$. Thus theoretical predictions to date support a transition to the CG structure
PACS numbers: 61.50.Ah, 61.50.Ks, 62.50.+p, 71.15.Mb

with no explanation for the large discrepancies with experiments.

In this Letter we report extensive first-principles simulations that reveal new structures and understanding of the complex behavior of $\mathrm{N}$. The present work is based upon consideration of a broader range of possibilities than previous studies, and upon improved generalized gradient approximation (GGA) density functionals. GGAs are essential for proper comparison of structures with such different bonding; this is well known from many other works, including various nitrogen chainlike molecules [16]. Results here use the Perdew-Burke-Ernzerhof (PBE)

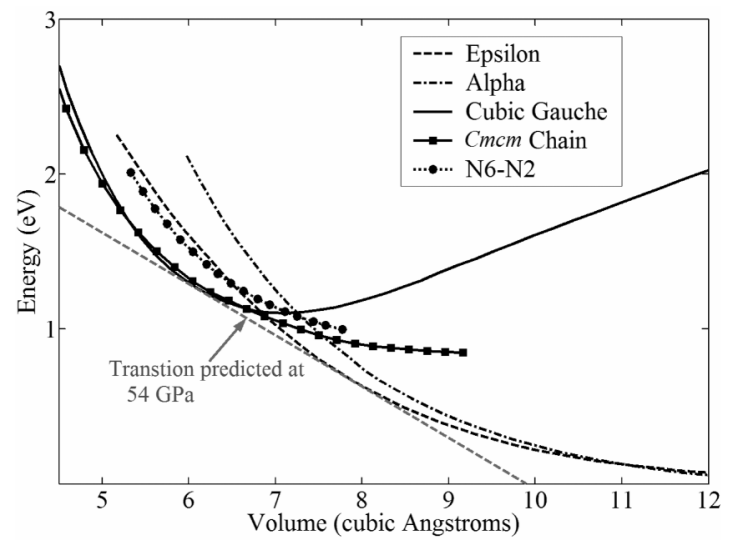

FIG. 1. Relative energies are shown as a function of volume of nitrogen in different structures. Two known molecular crystalline forms shown are: $\alpha$, a cubic $\mathrm{Pa} 3$ structure stable at low pressure, and $\varepsilon$, stable at higher pressure with a hexagonal structure shown in Fig. 2(b). "Cubic gauche" (CG) is the nonmolecular network form previously predicted [6] to be much more stable than any other nonmolecular form reported up to now [Fig. 2(d)]. The two new structures identified in the present work have energies given by the lines with symbols and the atomic structures are shown, respectively, in Figs. 2(a) and 2(c): a $\mathrm{Cmcm}$ chain structure that is energetically competitive with the $\mathrm{CG}$ structure and a hexagonal molecular form based on $\mathrm{N}_{2}$ and $\mathrm{N}_{6}$ units. The latter is never the energetically favored structure but may be observable due to large barriers to transitions to lower energy structures, as discussed in the text. 
form of the GGA [17] which has been shown to be accurate in many other examples, such as the phases of $\mathrm{SiO}_{2}$ under pressure [18]. The most striking new structures have been discovered using "first-principles" molecular dynamics simulations in which the atoms are moved thermally or allowed to relax and the electrons treated within density functional theory [11]. The calculations are done with the ab initio density functional code SIESTA [19] which uses an efficient local orbital basis that facilitates an extensive variety of thermal simulations and minimization with respect to the atomic positions and cell size and shape. The results were verified on final structures using the ABINIT [20] plane wave and the CRYSTAL [21] Gaussian codes.

In Fig. 1 are shown the final energies as a function of volume for the most relevant structures identified in the present work [22]. Two molecular phases are shown, $\alpha$ and $\varepsilon$; the latter is the known high pressure phase of $\mathrm{N}$ in agreement with our calculations. The transition to the CG phase is at a pressure of $\sim 54 \mathrm{GPa}$ (a volume change from 8-6 $\AA^{3}$ /atom as indicated by the tangent construction), in agreement with previous Monte Carlo calculations [15], supporting our present use of the PBE GGA functional over the local-density approximation functional which predicts a transition pressure of $\sim 26 \mathrm{GPa}$.

In order to search for possible structures, we initially carried out molecular dynamics simulations for cells of 64 atoms and high temperature (up to $10000 \mathrm{~K}$ ), and we identified characteristic structures by quenching. This led to mixtures of threefold coordinated atoms (like CG) and twofold coordinated atoms that are chainlike. The mixtures were history dependent indicating large energy barriers and competing structures.

Further studies at low temperature utilized simulations with various starting structures. The most striking new results have been found by thermal simulations and minimizations starting from the known $\varepsilon \mathrm{N}_{2}$ structure, which has a distorted hexagonal structure [Fig. 2(b)]. Because of a large energy barrier to formation of the CG structure [Fig. 2(d)], a transition to CG has not been observed in any of the simulations. However, the $\varepsilon$ structure was found to transform to two very different structures under different conditions. If the low pressure $\varepsilon$ structure is subjected to a sudden change in volume, through a homogeneous scaling of atomic and cell coordinates, it becomes unstable at $6.4 \AA^{3}$ /atom (or $\sim 60 \mathrm{GPa}$ final pressure) and transforms with no barrier to a hexagonal structure consisting of $\mathrm{N}_{2}$ and $\mathrm{N}_{6}$ molecules [Fig. 2(c)]. Even though the energy of $\mathrm{N}_{2}-\mathrm{N}_{6}$ is higher than the CG structure as shown in Fig. 1, this result shows that it may be created by appropriate conditions.

A new metallic chain phase is formed if the simulation is done following a more physical path in which the $\varepsilon$ structure is gradually compressed by increasing the pressure in increments of $10 \mathrm{GPa}$ and relaxing the cell at each

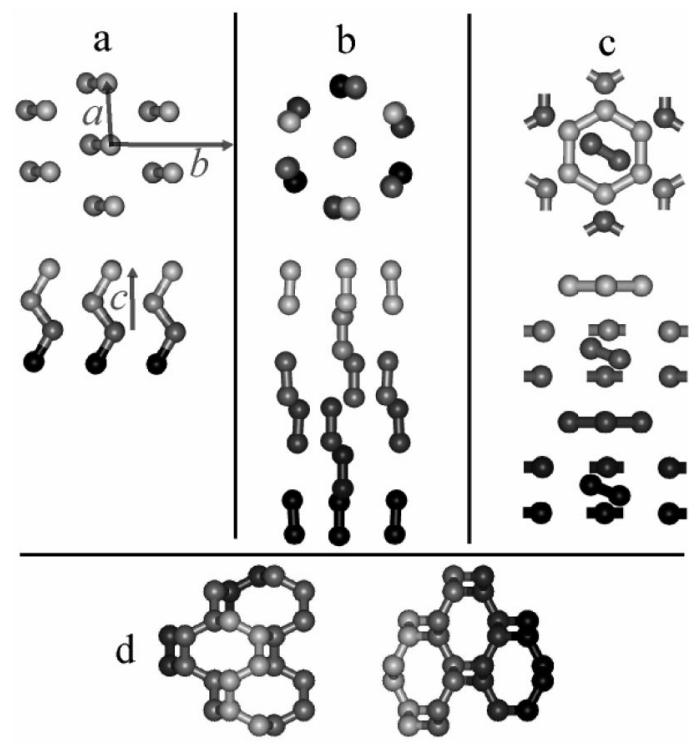

FIG. 2. From the left the structures are (a) the $\mathrm{Cmcm}$ chain, (b) the ideal molecular $\varepsilon$, (c) the $\mathrm{N}_{2}-\mathrm{N}_{6}$ structures, and (d) Cubic gauche. The upper part of (a) is a projection normal to the $c$ axis and the lower part is an orthogonal projection in a plane parallel to the $b$ and $c$ axes. The other panels are laid out similarly. For the transition from the $\varepsilon$ structure to the $\mathrm{N}_{2}-\mathrm{N}_{6}$ structure, three molecules rotate and bond to form a hexagon; the molecules in the center of the projection remain diatomic. In the transition to the $\mathrm{Cmcm}$ chain structure, molecules join with other molecules that are roughly in the same column, so that each of the seven groups seen in the $c$-axis projection of the $\varepsilon$ structure forms a chain.

pressure. In this case the $\varepsilon$ structure remains metastable up to much higher pressures until a point where the pressure is released through a spontaneous internal transformation to a base-centered orthorhombic chain structure (space group $\mathrm{Cmcm}$ ). This occurs at a final pressure of $\sim 220 \mathrm{GPa}\left(4.6 \AA^{3} /\right.$ atom $)$. Although this is far above the equilibrium transition pressure predicted (see Fig. 1), it is consistent with the overpressure of $\sim 200 \mathrm{GPa}$ required to cause a transition in recent experiments [1214]. As shown in Fig. 1, the $\mathrm{Cmcm}$ chain structure is essentially degenerate in energy with the network CG structure at high pressure, and at low pressures it is the lowest energy nonmolecular structure found to date. Since this chain structure is a stable minimum (at least metastable and possibly the global minimum for a range of pressures) and it is separated from the CG structure by a large barrier, the transition from $\varepsilon$ to the chain structure may be the most favorable kinetically allowed pathway for an actual transition.

Each chain has the zigzag structure shown in Fig. 2(a). This is the expected structure of an isolated nitrogen chain which is analogous to polyacetylene and has been studied theoretically [23]. The chains are very similar to those found in Ref. [8], but the different packing leads to a significantly lower energy $(\sim 0.18 \mathrm{eV} /$ atom lower at a 
volume of $6.45 \AA^{3}$ /atom in our calculations with the same GGA functional). The chains are oriented parallel to the $c$ axis and bend back and forth in planes perpendicular to the $a$ axis. The primitive cell contains two atoms and the lattice vectors are distorted simple hexagonal vectors: $(-a / 2, b / 2,0),(a / 2, b / 2,0),(0,0, c)$. The Cartesian atomic coordinates can be written as $\pm(0, w / 2, c / 4)$, where $w$ is the chain width and $d=\sqrt{w^{2}+c^{2} / 4}$ is the bond length. A geometry optimization carried out at the constant atomic volume of $6.95 \AA^{3}$ leads to $d=1.31 \AA$ and a bond angle of $114^{\circ}$. For this volume the value of $c$ is $2.203 \AA$, the optimal $a / c$ and $b / c$ ratios are, respectively, 1.15 and 2.26, and $w / c=0.324$ or $w / b=0.143$.

Further simulations provided tests of stability at low pressure and finite temperature. First we displaced each atom in a 64 atom cell randomly by a maximum of $1 \%$ or $5 \%$ of the bond length in each Cartesian direction and performed a conjugate gradient minimization on the randomly displaced system with a target pressure of 100 bars. The second test was to displace each atom along the bond with one of its neighbors, i.e., a Peierls distortion. A favored bond was chosen for each atom in the cell and that bond length was decreased by $2 \%$ or $10 \%$, then the same minimization was performed for both tests. The results of both types of tests were that the structure returned to the original hexagonally packed chain structure. The displacements increase the energy by up to $0.6 \mathrm{eV}$ per atom, indicating a substantial barrier to transition in the two directions that we searched. The chain structure is very stable up to pressures $\sim 800 \mathrm{GPa}$, above which it transforms into a fully connected structure with each atom being bonded to three others.

The electronic bands of the $\mathrm{Cmcm}$ chain structure at $25 \mathrm{GPa}\left(6.9 \AA^{3} /\right.$ atom $)$ are shown in Fig. 3, compared with the bands of a hypothetical isolated chain with the same internal coordinates. For the isolated chain the results are similar to those of Pohl et al. [23] but differ in detail due to the difference in bond angle. The two bands at the Fermi energy have, respectively, $\pi$ and $\sigma$ "lone-pair" character. Because of the extremely strong, short bonds, there is large dispersion along the chain; in addition, the chains have significant overlap as indicated by the dispersion of the bands perpendicular to the chains; this is shown clearly for $k$ vectors in the closed path from $\Gamma$ to $\Gamma$ at the left side of Fig. 3. For an isolated chain, two bands cross the Fermi energy leading to multiple Fermi "points" and a large density of states due to the flat band at $E_{\text {Fermi }}$. The bands of the solid are similar, but the dispersion perpendicular to the chains leads to large multiple parts of the Fermi surface in the Brillouin zone. This behavior is very different from graphite which has a pointlike Fermi surface made from $\pi$ bands, and it is similar to $\mathrm{MgB}_{2}$ which has multiple pieces of the Fermi surface with $\pi$ and $\sigma$ characters [24]. At higher pressures up to $\sim 200 \mathrm{GPa}$ the bands change gradually and have increased dispersion with features that should not be sensitive to the detailed approximations.

Nitrogen is expected to be an interesting metal. In particular, there are extremely high phonon frequencies and possible large electron-phonon coupling in the chain structure. The coupling may not be as strong as $\mathrm{MgB}_{2}$ since the $\sigma$ state has primarily nonbonding character in the $\mathrm{N}$ chain; however, there may be increased coupling due to the proximity to a Peierls transition. While this work provides no direct evidence for superconductivity, the analogy with known superconductors is suggestive, and the fact that $\mathrm{N}$ exhibits metastability [12-14] may open the possibility for superconducting nitrogen at low (or even zero) pressure.

There is other evidence that nitrogen can occur in chain structures. Density-functional theory and coupled cluster studies [16] of stable poly-nitrogen molecular structures reveal structural similarity between $\mathrm{N}_{10}$ chains and our Cmma structure. From this and the stability tests mentioned above, it is expected that a packed chain solid will

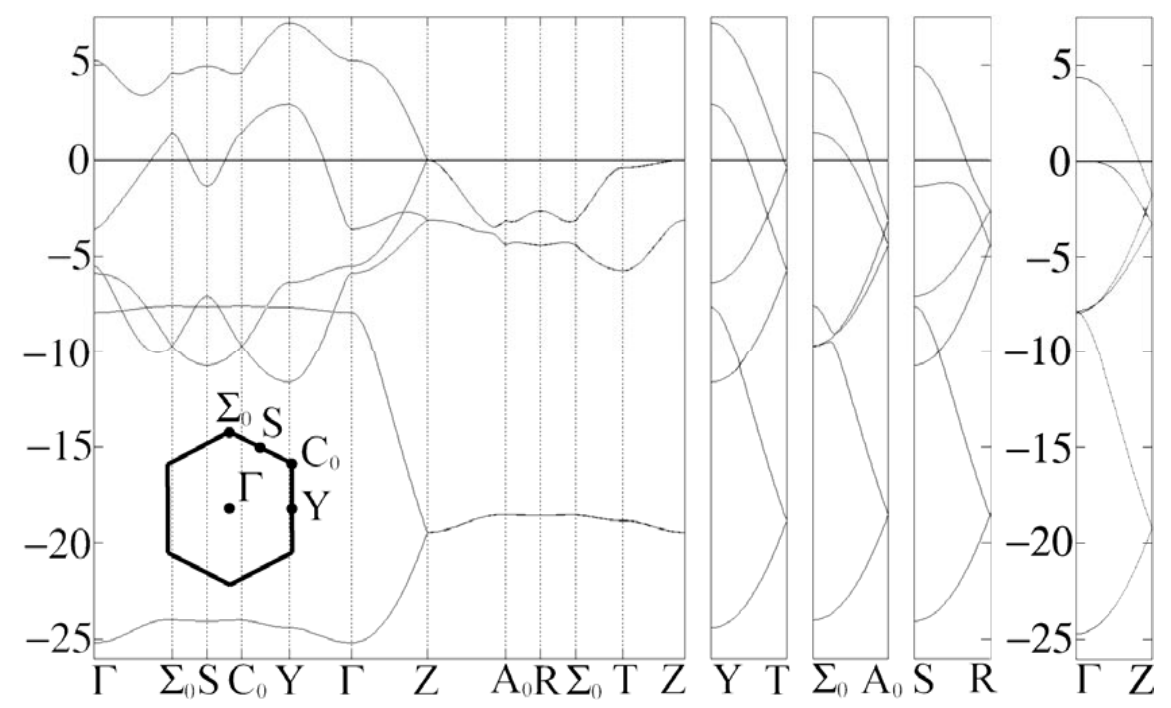

FIG. 3. The band structure of the $\mathrm{Cmcm}$ chain structure at $25 \mathrm{GPa}$ (four frames on the left) compared to a single isolated chain with the same internal chain coordinates (right frame). The inset shows the points in the plane perpendicular to the chain axis (the $c$ axis which is the [001] direction) containing the $\Gamma$ point; the path $Z-A_{0}-R-\Sigma_{0}-T-Z$ is on the face of the Brillouin zone with $k_{z}=\pi / c$. Dispersion along the chain direction is shown by the lines $\Gamma-Z$, $Y-T, \Sigma_{0}-A_{0}$, and $S-R$. The interchain coupling leads to differences from the isolated chain, including multiple crossings of the Fermi energy and a large Fermi surface with three-dimensional character. 
be stable at some high pressure and perhaps metastable over long time periods at near ambient pressure. It is also worth noting that other elemental diatomic molecules form low-energy chain structures: under pressure $\mathrm{Cl}$, $\mathrm{Br}$, and I [25,26] are all known to undergo phase transitions to chainlike structures. Metallic chains also appear to be important in the simulations of hydrogen [27] and the liquid phase of carbon between 2500 and $3000 \mathrm{~K}[28]$.

Finally, the present work provides a natural explanation for recent experiments [12-14] that have found hysteresis and metastability at low temperature. Large hysteresis is common for covalent solids [29]. We find that $\mathrm{N}$, a neighbor of $\mathrm{C}$ in the periodic table, acts in a manner similar to $\mathrm{C}$ at high pressures with multiple bonding and barriers making such structures metastable (like diamond) with long lifetimes even at zero pressure, as suggested by recent experiments [13]. Metastability may open the possibility for many forms including strong insulators and metallic superconducting nitrogen at relatively low (or even zero) pressure.

We gratefully acknowledge funding from the Center for Simulation of Advanced Rockets at the University of Illinois Urbana-Champaign, supported by the U.S. DOE through the University of California, Subcontract No. B523819. The work was also supported by the DOD MURI Grant No. AA-5-7232-A1 managed by the Army Research Office. Computational support was provided by the Materials Computation Center and the National Center for Supercomputing Applications at the University of Illinois, and the Major Shared Research Center of the Army Research Laboratory. D. S.-P. acknowledges support from Spanish MCyT under the "Ramon y Cajal" program.

*Present address: U.S. Army Research Laboratory, Aberdeen Proving Ground, MD 21005-5066, USA.

[1] W. J. Nellis, H. B. Radousky, D. C. Hamilton, A. C. Mitchell, N. C. Holmes, K. B. Christianson, and M. van Thiel, J. Chem. Phys. 94, 2244 (1991).

[2] D. C. Hamilton and F. H. Ree, J. Chem. Phys. 90, 4972 (1989).

[3] R. M. Martin and R. J. Needs, Phys. Rev. B 34, 5082 (1986).

[4] A. K. McMahan, Physica (Amsterdam) 139\&140B+C, 31 (1986).

[5] S. P. Lewis and M. L. Cohen, Phys. Rev. B 46, 11117 (1992).

[6] C. Mailhiot, L. H. Yang, and A. K. McMahan, Phys. Rev. B 46, 14419 (1992).

[7] J. D. Kress, S. Mazevet, L. A. Collins, and W.W. Wood, Phys. Rev. B 63, 24203 (2000); L. E. Fried and W. M.
Howard, J. Chem. Phys. 109, 7338 ( 1998), and references therein.

[8] M. M. G. Alemany and J. L. Martins, Phys. Rev. B 68, 024110 (2003).

[9] H. Olijnyk and A. P. Jephcoat, Phys. Rev. Lett. 83, 332 (1999).

[10] A. Mujica, A. Rubio, A. Muñoz, and R. J. Needs, Rev. Mod. Phys. 75, 863 (2003).

[11] R. M. Martin, Electronic Structure: Basic Theory and Practical Methods (Cambridge University Press, Cambridge, 2004).

[12] A. F. Goncharov, E. Gregoryanz, H. K. Mao, Z. Liu, and R. J. Hemley, Phys. Rev. Lett. 85, 1262 (2000).

[13] M. I. Eremets, R. J. Hemley, H. K. Mao, and E. Gregoryanz, Nature (London) 411, 170 (2001).

[14] E. Gregoryanz, A. F. Goncharov, R. J. Hemley, and H. K. Mao, Phys. Rev. B 66, 224108 (2002).

[15] L. Mitas and R. M. Martin, Phys. Rev. Lett. 72, 2438 (1994).

[16] R. J. Bartlett, S. Fau, M. Tobita, K. Wilson, and A. Perera (to be published).

[17] J. P. Perdew, K. Burke, and M. Ernzerhof, Phys. Rev. Lett. 77, 3865 (1996); 78, 1396(E) (1997).

[18] D. R. Hamann, Phys. Rev. Lett. 76, 660 (1996).

[19] J. M. Soler, E. Artacho, J. D. Gale, A. Garcia, J. Junquera, P. Ordejon, and D. Sanchez-Portal, J. Phys. Condens. Matter 14, 2745 (2002).

[20] A. Gonze, J. M. Beuken, R. Caracas, F. Detraux, M. Fuchs, G.-M. Rignanese, L. Sindic, M. Verstraete, G. Zerah, F. Jollet, M. Torrent, A. Roy, M. Mikami, Ph. Ghosez, J. Y. Raty, and D. C. Allan, Comput. Mater. Sci. 25, 478 (2002).

[21] V. R. Saunders, R. Dovesi, C. Roetti, M. Causà, N. M. Harrison, R. Orlando, and C. M. Zicovich-Wilson, CRYSTAL98 User's Manual (University of Torino, Torino, 1998).

[22] Energy differences were converged to less than $0.01 \mathrm{eV} /$ atom using regular grids of $k$ points. For example, the $\mathrm{Cmcm}$ structure was treated with a 16-atom cell (for comparison with other structures) and a $4 \times 4 \times$ 4 grid, corresponding to 512 points in the Brillouin zone of the primitive 2-atom cell.

[23] A. Pohl, H. Meider, and M. Springborg, J. Mol. Struct., Theochem 305, 165 (1994).

[24] J. M. An and W. E. Pickett, Phys. Rev. Lett. 86, 4366 (2001).

[25] N. H. March and M. P. Tosi, Phys. Chem. Liq. 35, 131 (1997).

[26] H. G. Drickamer, Solid State Phys. 17, 1 (1965); H. G. Drickamer, R.W. Lynch, R. L. Clendenen, and L. PerezAlbuerne, Solid State Phys. 19, 135 (1966).

[27] M. Ross and L. H. Yang, Phys. Rev. B 64, 134210 (2001), and references cited therein.

[28] G. Galli, R. M. Martin, R. Car, and M. Parrinello, Phys. Rev. B 42, 7470 (1990).

[29] See, for example, Fig. 10 in F. P. Bundy, J. Geophys. Res. 85, 6930 (1980). 